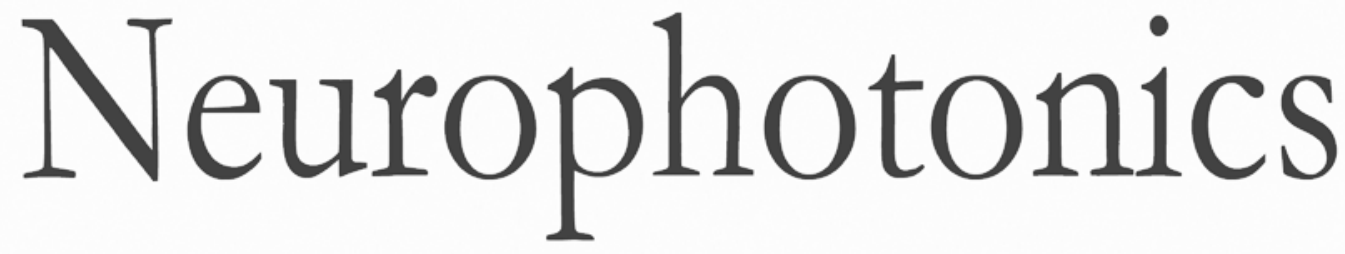

\title{
Methodology for high-yield acquisition of functional near-infrared spectroscopy data from alert, upright infants
}

James R. Goodwin

Ashley E. Cannaday

Holly G. Palmeri

Aldo Di Costanzo

Lauren L. Emberson

Richard N. Aslin

Andrew J. Berger 


\title{
Methodology for high-yield acquisition of functional near-infrared spectroscopy data from alert, upright infants
}

\author{
James R. Goodwin, ${ }^{a, b, *, \dagger}$ Ashley E. Cannaday, ${ }^{a, \dagger}$ Holly G. Palmeri, ${ }^{c}$ Aldo Di Costanzo, ${ }^{a, d}$ Lauren L. Emberson, ${ }^{c}$ \\ Richard N. Aslin, ${ }^{c}$ and Andrew J. Berger ${ }^{a, e}$ \\ aUniversity of Rochester, Institute of Optics, Rochester, New York 14627, United States \\ ${ }^{\mathrm{b}}$ Queensland University of Technology, School of Chemistry, Physics and Mechanical Engineering, Brisbane, Queensland 4001, Australia \\ 'University of Rochester, Department of Brain and Cognitive Sciences, Rochester, New York 14627, United States \\ dUniversidad Autónoma de Nuevo León, Facultad de Ciencias Físico Matemáticas, Avenida Universidad s/n, Cd. Universitaria, \\ San Nicolás de los Garza, Nuevo León 66455, México \\ eUniversity of Rochester, Department of Biomedical Engineering, Rochester, New York 14627, United States
}

\begin{abstract}
Functional near-infrared spectroscopy (fNIRS) research to date has tended to publish group-averaged rather than individual infant data due to normative basic research goals. Acquisition of individual infant time courses holds interest, however, both for cognitive science and particularly for clinical applications. Infants are more difficult to study than adults as they cannot be instructed to remain still. In addressing this, upright infants pose several associated complications for the researcher. We identified and optimized the factors that affect the quality of fNIRS data from individual 6- to 9-month-old infants exposed to a visual stimulation paradigm. The fNIRS headpiece was reconfigured to reduce inertia, increase comfort, and improve conformity to the head, while preserving fiber density to avoid missing the visual cortex activation. The visual-stimulation protocol was modified to keep the attention of infants throughout the measurement, thus helping to reduce motion artifacts. Adequate optical contact was verified by checking power levels before each measurement. By revising our experimental process and our data rejection criteria to prioritize good optical contact, we report for the first time usable hemodynamic data from $83 \%$ of infants and that two-thirds of infants produced a statistically significant fNIRS response. @ 2016 Society of Photo-Optical Instrumentation Engineers (SPIE) [DOI: 10.1117/1.NPh.3.3.031415]
\end{abstract}

Keywords: functional near-infrared spectroscopy; hemodynamics; brain activation; visual cortex; clinical; heartbeat.

Paper 15065SSRR received Dec. 31, 2015; accepted for publication Jul. 6, 2016; published online Jul. 28, 2016.

\section{Introduction}

Infants' cerebral hemodynamics have been studied using nearinfrared light for more than 18 years, with the first publication by Meek et al. ${ }^{1}$ in 1998. Notable reviews by Lloyd-Fox et al. ${ }^{2}$ covering the time frame from the first publication to April 2009 and an online database by Cristia et al. ${ }^{3}$ reviewing the literature to July 2012 indicate that there were 76 articles and theses, which report on 109 different studies across a range of age groups. There are two major infant groupings according to Cristia et al. ${ }^{3}$ that have been most commonly studied: newborns and 4- to 8-month-old infants. Studies by other groups, ${ }^{4-10}$ in the age range of 5 to 7 months similar to the age range in our study, focused on the occipital, temporal, and parietal cortices.

There is considerable interest in using functional near-infared spectroscopy (fNIRS) to study infants with more recent studies (since July 2012) covering its broad potential as a noninvasive tool to study infant cognition, ${ }^{10}$ typical and atypical development, ${ }^{11}$ and, more specifically, to study face recognition, ${ }^{12-14}$ shape processing, and motion ${ }^{15,16}$ and color priming. ${ }^{17}$ Despite the rapid growth in the number of infant fNIRS publications in recent years, this number is relatively low when compared to that of adult studies. ${ }^{18}$ Infants are certainly more difficult to

*Address all correspondence to: James R. Goodwin, E-mail: james.goodwin@ rochester.edu

†The first two authors contributed equally to this work. study, as they cannot be instructed to remain still, unlike an adult subject. Upright infants pose several complications for the researcher in obtaining good quality fNIRS data. Analysis of infant studies of all age groupings by Cristia et al. ${ }^{3}$ and Lloyd-Fox et al. ${ }^{2}$ reveals that $\sim 40 \%$ of data (at the infant level) is rejected. This high attrition rate is usually attributed to low signal-to-noise or less compliant infant subjects, which can be specifically due to a range of factors such as motion artifacts, lack of optical contact due to hair obstruction, improper fit of the NIRS cap, fussiness, or inattentiveness.

However, most studies do not address these factors, and, to our knowledge, the necessary protocol modifications to reduce the effect of these factors and to acquire good quality data from upright, alert infants have not been explicitly outlined in the literature. In this study, we present the steps taken to improve the probe design and placement, stimulus and rest protocols, and optical contact. In addition, we present the data yield results for the analysis of infant subjects based on their individual time courses.

Unlike other infant fNIRS research that asks developmental questions and averages group data from subjects in the study, this study focuses on protocols for high-yield data acquisition and analysis of data at the single-infant level. As a result, we ultimately determine the percentages of infants that yield usable hemodynamic data (based on heartbeat and motion 
artifact criteria), and of these, the percentage of infants whose response to the visual stimulus is flagged as an activation. This type of information is important in both research and clinical environments.

\section{Methods}

\subsection{Instrumentation and Probe Design}

The fNIRS system used in this study (Cephalogics LLC, Boston, Massachusetts; for details, see Ref. 19) utilized nearinfrared LED light delivered via optical fiber bundles at two wavelengths (750 and $850 \mathrm{~nm})$. The instrument houses a total of 24 sources and 21 detectors $(24 \times 21)$ with an average laser power level of $200 \mu \mathrm{W}$ at each wavelength for each source. $^{19}$

A study across all infant fNIRS research (to 2012) by Cristia et al. ${ }^{3}$ investigating the attrition rate (infant exclusion) as a function of the total number of sources and detectors (optodes) notes that using fewer than 20 fiber bundles results in a significant reduction in the number of infants excluded. This aligns with our own experience in which larger, bulkier probes fail to maintain consistent optical contact during infant motion, causing severe artifacts. Lightweight probes are also easier to affix quickly to the infant's head, thereby saving more of the infant's limited attention span for data acquisition.

In keeping with a lightweight yet high density design, we constructed a 14-fiber array (six sources and eight detectors) from a subset of the commercial instrument's fiber bundles. A harness supporting the fiber cables was suspended from the ceiling above, further reducing the weight on the infant's head. While the majority of each bundle is flexible, it terminates in a rigid tip that makes a right angle bend at $\sim 14 \mathrm{~mm}$ from the end. These tips were inserted directly into a customized pad containing a triangular array of holes to accept the fibers, as shown in Fig. 1(a).

The pad consisted of two layers of commonly available materials, ethylene vinyl acetate (EVA) foam $(6 \mathrm{~mm})$ and silicone rubber $(6 \mathrm{~mm})$, thus $\sim 12 \mathrm{~mm}$ in total thickness. The EVA foam is commonly used in gym flooring and was sliced from an original thickness of 12 to $6 \mathrm{~mm}$. The silicone used in the design was a high temperature silicone that is typically used for cooking applications. Both materials were obtained from retail stores in the color black, thus reducing stray light leakage from sources to detectors.

The layers of material work in a complementary fashion, in that the silicone layer provides a firm but flexible substrate to securely hold the optical fiber while the foam layer provides stability for the optical fibers at the right angle to minimize any potential leverage force, which could result in potential movement at the fiber tip against the head surface. When inserted, the fiber tips were flush with one surface of the pad (to contact the head), while the 90-deg bend occurred just above the other surface (to allow the adjacent portion of the fiber bundle to lie flat), as shown in Fig. 1(c). The probe was secured to the infant's head by two mesh head bands, as shown in Fig. 1(b). The presence of hair in general did not cause problems in acquiring data. As the average age of the infants was less than 7 months, typically the hair was not long, thick, or dark enough to be obstructive of the light delivered to the scalp.

The triangular grid of fibers consisted of nearest-neighbor spacings (between source and detector) of 10, 22, 30, and $36 \mathrm{~mm}$ with 18, 16, 4, and 6 channels, respectively, of each separation distance. In this study, we analyzed all of the channels and grouped our analysis to include the 22- and 30-mm channels

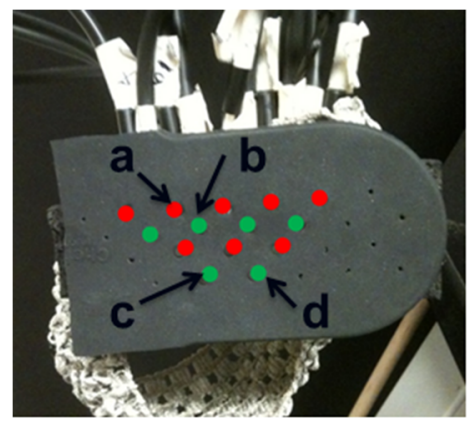

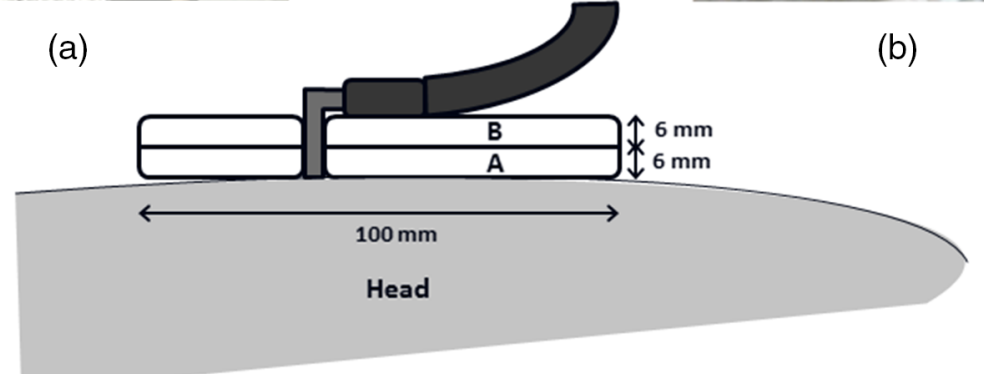

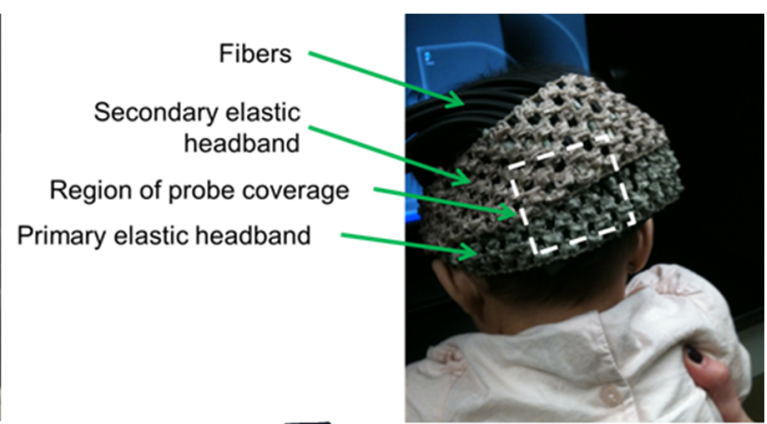

(b)

(c)

Fig. 1 (a) Infant probe: photograph of the surface of our infant probe showing sources (red circles) and detectors (green circles). An example of each of the separation distances between the source and detector of the first nearest neighbor ( $a$ to $b=10 \mathrm{~mm}$ ), second nearest neighbor ( $a$ to $c=22 \mathrm{~mm}$ ), and third nearest neighbor ( $a$ to $d=30 \mathrm{~mm}$ ) is shown. (b) Probe placement: photograph of an infant subject showing the probe placement, which is underneath both primary and secondary elastic headbands. (C) Probe cross section: schematic diagram of the cross-section of the infant probe showing optical fiber supported by the two complementary layers of material $(A)$ silicone and $(B)$ foam rubber. 

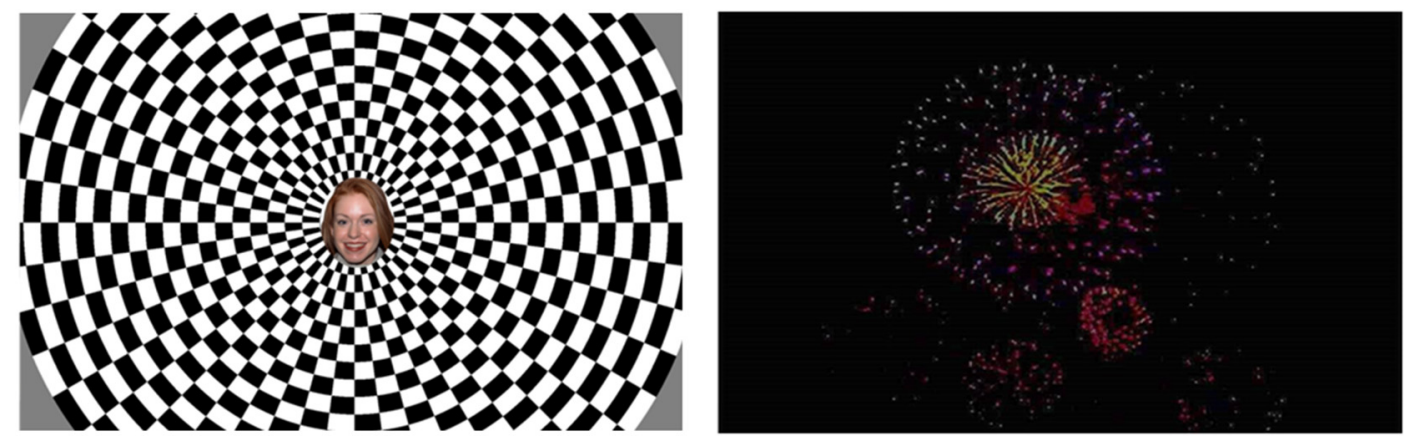

Fig. 2 Images from the video display during stimulus and rest conditions. During the stimulus interval, the reversing checkerboard cycles at a rate of $3.4 \mathrm{~Hz}$, while the face in the middle "looms" (grows larger and smaller) and changes to a new face every two cycles. The rest period features a dim fireworks animation.

(a total of 20), as they provide ample sampling of the outer cortex for this age group, as established by Taga et al. ${ }^{20}$ and Gervain et al. ${ }^{21}$

\subsection{Subjects}

Fifty-four healthy subjects (mean age: 6.8 months, age range: 6.2 to 8.3 months, 26 females) participated in this study, which was approved by the University of Rochester Research Subjects Review Board. The mean age is skewed toward the lower end of the range because over time, we found infants less than 7 months old to be more compliant with our particular stimulation protocol. Infant subjects were consented into the study via their parent or guardian. During measurements, the adult and infant were seated with the infant sitting on the parent's lap approximately 18 " to 24 " from a computer screen that presented the visual stimulation trial, as described below.

\subsection{Visual Stimulation Paradigm}

The measurement trials followed a standard block design with alternating visual stimulus and rest periods. Soft calming nursery music ["Camptown Races" (Baby Music, album released 2010)] was played in the background throughout each trial.

\subsubsection{Visual stimulus}

As shown in Fig. 2, the stimulus condition consisted of a full (360 deg) radial checkerboard pattern with a face at the center selected at random and chosen from a group of predetermined faces from the "NimStim Set of Facial Expressions."22 The face grows and shrinks in size (looms) ${ }^{23}$ from $\sim 0.5$ to $2.6 \mathrm{~cm}$ and changes to a new face every two cycles to hold the infant's attention.

The reversing checkerboard stimulus was designed to activate the occipital cortex cycles at a rate of $\sim 3.4 \mathrm{~Hz}$. The checkerboard flashes for $7 \mathrm{~s}$, which is a balance between allowing sufficient time for a cortical response and providing as many trials as possible within a relatively short time frame to keep the infant's attention.

\subsubsection{Rest interval}

The rest condition consisted of $7 \mathrm{~s}$ of a dim movie of fireworks (Fig. 2) ${ }^{6}$ followed by looming faces (serving as a cue for the experimenter to advance to the next trial). That is, the rest condition consisted of $7 \mathrm{~s}$ of a dim movie plus looming faces, which was typically 1 to $3 \mathrm{~s}$ (or more) and controlled by the experimenter. Therefore, the total time of the rest condition was anywhere between $8 \mathrm{~s}$ (at the minimum) and $10 \mathrm{~s}$ (more typical). The rest condition is calm enough to contrast with the stimulus condition but engaging enough to keep the infant focused, and thus minimizes motion artifacts between trials. The onset of the next trial (cued by looming faces) is triggered via a key stroke, allowing the experimenter control in real time. The length of the rest condition is therefore dependent on whether the infant subject is starting to lose interest or needs extra time for refocusing. The experimenter pressed the key as soon as the infant appeared to be looking at the screen or if approximately $3 \mathrm{~s}$ had elapsed. In the latter case, the onset of the flashing checkerboard stimulus in an otherwise dark room often caused the infant to return his or her gaze to the screen. The varying time (usually 8 to $10 \mathrm{~s}$ ) allows for a flexible compromise between the minimum necessary rest period needed to facilitate the return to baseline hemodynamics and a maximum time parameter to enable continued engagement of the baby's attention.

\subsubsection{Infants, runs, and trials}

Data were collected from all of the 54 infants, with each participant sitting for either one or two measurement runs. About 25 of the 54 infants sat for two consecutive measurement runs (and thus 29 sat for one run) consisting of a maximum programmed seven trials in each. The trials were repeated until the infant became unresponsive, however, each infant usually sat through the maximum seven trials. Each trial consisted of a stimulus and rest interval (as described). The total number of trials for all infants across all measurement runs was 534, with a mean of 9.89 per infant. These and other key parameters are summarized in Table 1.

\subsection{Probe Placement}

\subsubsection{Accuracy in probe placement}

The probe was placed over the occipital lobe in each of the 54 infants, using the inion as a fiducial marker on the scalp. The center of the probe was placed 2 to $3 \mathrm{~cm}$ above the inion. We learned that probe placement needs to be at a higher relative distance above the inion than usually would be undertaken compared to the corresponding scalp landmarks for an adult subject. That is, as reported by Kabdebon et al. ${ }^{24}$ in a study correlating anatomical structures with sensor placements, infants' 
Table 1 Values of key parameters from the infant visual stimulation study.

\begin{tabular}{lc} 
Parameter & Value \\
\hline Infants & 54 \\
Runs & 79 \\
Trials & 534 \\
Mean ( \pm s.d.) trials per infant & $9.89( \pm 0.62)$ \\
Minimum and maximum trials for any one infant & 3 and 14 \\
\hline
\end{tabular}

landmarks project over lower brain regions (occipital and temporal) than adults'.

\subsubsection{Ensuring optical contact}

Placing fiber probes on infants' heads securely, with minimal influence from hair, is important for NIRS recordings. Checking the quality of optical contact prior to data acquisition can be done in various ways. If a NIRS instrument displays realtime strip charts of optical data, one can look for evidence of heartbeats after attaching the probe. Heartbeat pulses, in our experience, are typically $3-5 \mathrm{X}$ weaker in amplitude than visually evoked hemodynamic responses measured with 22-mm sourcedetector separations. As such, visibility of heartbeats after probe attachment implies that the optical conditions are suitable for detecting visual responses to a subsequent stimulus protocol. If heartbeats are not visible due to low signal to noise, the probe can be adjusted in hopes of improving contact.

Because the Cephalogics instrument displayed only a running average (over a few seconds) of the power in each channel, direct observation of heartbeats was not possible. An indirect estimate was therefore devised, based upon the empirical observation that heartbeats in 22-mm channels typically modulate the detected 850 -nm optical power by $1 \%$. After securing the fiber headpiece, power readings from the 22-mm channels were therefore compared to the average of the unused (i.e., dark) channels of the instrument. If a $22-\mathrm{mm}$ channel was $100 \times$ stronger than the electronic noise floor, that meant that heartbeat pulses in that channel $(100 \times$ weaker than the total signal) should be visible above the noise in a time-resolved signal.

If a majority of the $22-\mathrm{mm}$ channels met this $100 \times$ criterion, optical contact was assumed to be sufficient. Otherwise, the probe was adjusted once in an attempt to improve contact and boost the detected power level. Adjustments happened approximately once out of every four data runs. It would typically take less than $1 \mathrm{~min}$ to secure the probe to the infant's head either for the first time or as an adjustment. This single adjustment step allowed us to increase our yield of usable data without signicantly extending the amount of time the infant had to spend wearing the probe. Anecdotally, we note that the adjustment usually increased the power; in future studies, the initial and adjusted power levels could be recorded in order to quantify how often this occurred.

\section{Data Analysis}

The analysis of the data first involved a preprocessing step of converting the detected power to concentrations of oxy- and deoxyhemoglobin. The time courses of each channel were then screened with two criteria, heartbeat and motion artifacts, with the channels passing both criteria defined as "analyzable channels."

\subsection{Data Preprocessing}

As described in previous work, ${ }^{25}$ the data were processed prior to screening for analyzable channels. Each time series of detected power at the two wavelengths $(750$ and $850 \mathrm{~nm})$ was converted to changes in optical density and subsequently to changes in oxy- and deoxyhemoglobin concentration. ${ }^{26}$

\subsection{Screening for Analyzable Channels}

fNIRS measurements are intended to sense hemodynamic activity in the volume optically explored. As noted in Sec. 1, however, low light levels and large motion artifacts can corrupt the data. We screened out data with poor optical contact and/or large motion artifacts using the steps described below.

\subsubsection{Heartbeat detection}

To judge whether recording conditions in a given fNIRS channel are good enough to detect visually evoked hemodynamics, one can use heartbeat pulses as an internal standard. The Cephalogics instrument acquires data at $10.8 \mathrm{~Hz}$, which is sufficient to resolve heartbeats in the oxyhemoglobin signal. As discussed above, in our experiments with infants, heartbeat pulsations measured at 22- and 30-mm separations are typically three to five times smaller in amplitude than visually evoked oxyhemoglobin surges. Empirically, then, the visibility of heartbeats establishes that the recording conditions are generally adequate for observing visual responses.

We Fourier-transformed each stimulus period's time series (including a post- and pretime of $2 \mathrm{~s}$ ) and ratioed the power under two equal-width frequency bands, one containing the infant heart rate $(1.75$ to $2.50 \mathrm{~Hz})$ and one at higher frequencies presumed to contain only noise $(3.0$ to $3.75 \mathrm{~Hz})$. The time course was rejected if the heart rate to noise ratio, averaged over all stimulus periods, was less than an empirically chosen threshold of 2.0.

The heartbeat threshold is routinely exceeded at 22 and $30 \mathrm{~mm}$. At the larger separation distance of $36 \mathrm{~mm}$, the detected optical power is usually so low that the electrical noise obscures the heartbeat. In particular, only about $11 \%$ of the $36-\mathrm{mm}$ channels pass the heartbeat standard compared to approximately (on average) $56 \%$ of the 22 - and $30-\mathrm{mm}$ channels. Consequently, in this study, we present data from the 22 and $30 \mathrm{~mm}$ distances grouped together. This lies within most of the range of separation distances cited in reviews $(\sim 93 \%)^{2,3}$ and also conforms to the distances recommended by Taga et al. ${ }^{20}$

\subsubsection{Motion artifact detection}

Infant head motion can cause changes in detected light levels that are distinguishable from hemodynamic effects in abruptness, amplitude, and degree of crosstalk between the calculated oxy- and deoxyhemoglobin concentration changes. Figure 3 shows examples of calculated oxyhemoglobin time courses containing artifactual spikes. Such artifacts need to be removed before further data analysis can be performed. For the present study, we developed an automated motion artifact detection technique based on similar approaches, as outlined by Brigadoi 


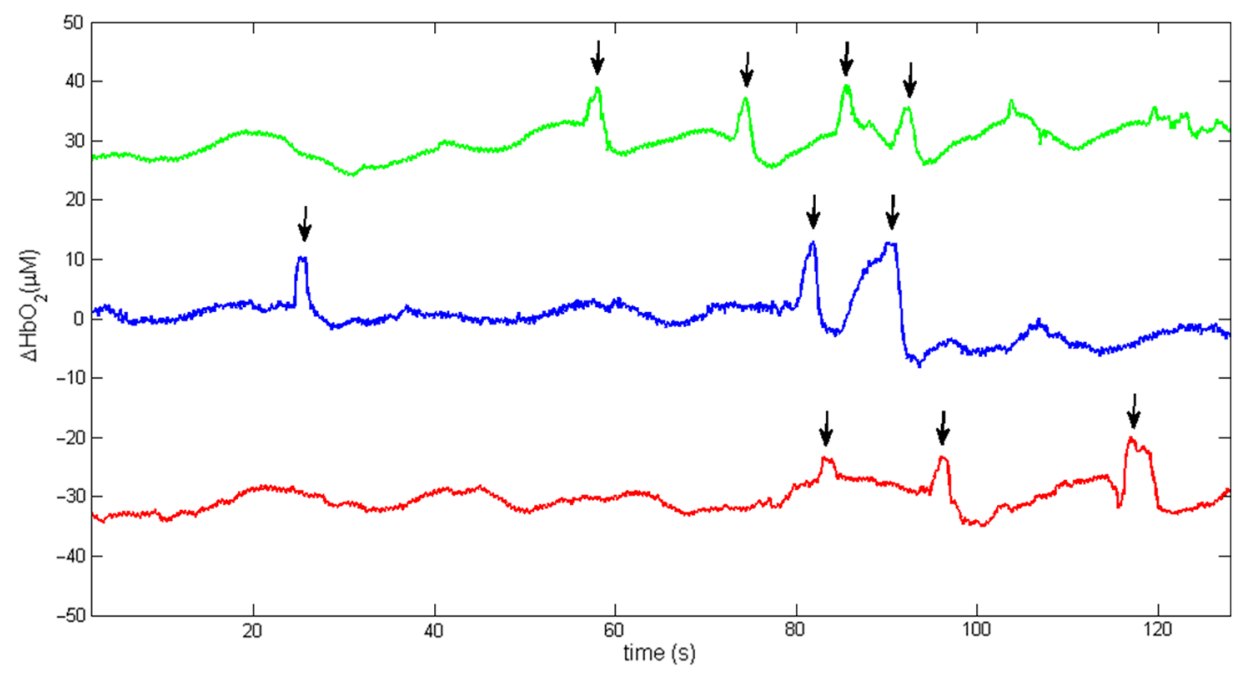

Fig. 3 Typical time courses showing motion artifacts identified by evaluators (marked by arrows). Channels were rejected and not analyzed any further unless at least two trials were artifact-free.

et al., ${ }^{27}$ who employed the Homer2 software for NIRS analysis ${ }^{28}$ within MATLAB (Mathworks, Massachusetts, United States).

Each time course was first high-pass filtered by subtracting a 25-s moving boxcar average. The amplitude change over a five time-point window $(\sim 0.5 \mathrm{~s})$ was compared to empirically selected thresholds of 2.0 and $3.55 \mu \mathrm{M}$ for the 22- and 30-mm channels, respectively. In particular, the difference in every fifth pair of time points is calculated in a moving window to establish a comparison with the threshold value. If this difference value was greater than the threshold value and located within the stimulus period (between the onset and $2 \mathrm{~s}$ after the conclusion of the stimulus period), then the artifact was flagged as motion and the trial subsequently rejected. Once all of the trials with motion were removed, the channels with at least two trials still remaining were retained for subsequent testing for potential activations.

\subsection{Flagging of Activations}

For each analyzable channel, we tested whether a hemodynamic activation was present in response to the visual stimulation. A channel was flagged if the average rise in oxyhemoglobin concentration over all of the stimulation periods was significantly positive, as previously utilized by Saager et al. ${ }^{29}$ and Goodwin et al. ${ }^{25}$ (Deoxyhemoglobin activation responses were regularly observed to be anticorrelated with those of oxyhemoglobin and routinely five times weaker. For simplicity, calculation of apparent activation response rates was performed here using oxyhemoglobin alone.) Specifically, the average value of the $\Delta \mathrm{HbO}_{2}$ level within a 2-s window, from the onset of an individual stimulus period, was subtracted from the average value within a 2-s window from the conclusion of that stimulus. A one-sided $t$-test checked whether the mean of the multiple values (across all of the trials) was greater than zero. This a priori reasoning is supported by the extensive database developed by Cristia et al., ${ }^{3}$ which lists all $100+$ infant studies up until 2012 and reports variables such as oxy- and deoxyhemoglobin levels and cortices studied. Essentially, all studies that involved a visual stimulus and that monitored the occipital cortex showed an increase in oxyhemoglobin levels.

The significance level for the $t$-test was set at 0.05 (95\%). For an infant to be flagged as an "activator," our criterion required that at least one channel (consisting of at least two trials ${ }^{30}$ ) per infant pass the $t$-test.

\section{Results}

\subsection{Selection of Analyzable Channels}

Table 2 summarizes the results of applying our selection criteria to data acquired from 54 infants. As mentioned, the 22- and 30-mm channels (a count of 16 and 4, respectively) have been grouped together and presented in this analysis because they have a similar proportion ( $61 \%$ and $57 \%$, respectively) that

Table 2 Summary of results: number and percentages of channels and infants that pass each criterion.

\begin{tabular}{|c|c|c|c|c|}
\hline Criteria & & $\begin{array}{c}\text { Number of channels } \\
(22 \text { and } 30 \mathrm{~mm})\end{array}$ & Number of infants & $\begin{array}{l}\text { Proportion of infants } \\
\text { (relative to previous step) }\end{array}$ \\
\hline All data & & 1580 & 54 & $\mathrm{n} / \mathrm{a}$ \\
\hline \multirow{2}{*}{$\begin{array}{l}\text { Criteria for accepting channels } \\
\text { as "analyzable" }\end{array}$} & (a) Heartbeat detected & 922 & 54 & 100 \\
\hline & $\begin{array}{l}\text { (b) At least two trials not affected by } \\
\text { motion artifacts (per channel) }\end{array}$ & 546 & 45 & 83 \\
\hline Activation flagged by $t$-test & & 166 & 31 & 69 \\
\hline
\end{tabular}


passes the heartbeat metric and lies within the range of typical separation distances used in most studies.

In the compilation of Table 2, total numbers of channels (analyzable or activating) were calculated without taking the relative position of the channels into account. Fundamentally, one would expect all of the activation-flagged channels to form contiguous regions, and contiguity might be used as a criterion to reject activation labels for spatial outliers. A spatial analysis of this sort proved inconclusive on this dataset (perhaps due to the imprecise task of defining activations) but would be a desirable future approach.

The inclusion of only the 22- and 30-mm channels resulted in a total of 1580 of such channels (i.e., 20 total channels times 79 data runs; see Table 1). About 922 of the 1580 inspected, representing $58 \%$, met the first criterion of a detectable heartbeat. This subset included channels from all of the 54 infants. Of the 922 channels that exhibited heartbeat, 546 (59\%) contained at least two trials not affected by motion artifacts. This subset included channels from 45 of the 54 infants.

Overall, heartbeats were observed in $100 \%$ of the infant subjects and analyzable channels were obtained from $83 \%$ of the initial cohort. Only nine subjects had all of their channels rejected on basic grounds of poor optical contact and/or excessive motion artifacts. On average, 12 channels per infant passed the two criteria of heartbeat and at least two trials not affected by motion.

\subsection{Stimulus-Correlated Hemodynamics}

About 166 of the 546 analyzable channels (30\%) exhibited a significant stimulus-correlated rise in oxyhemoglobin concentration using the $t$-test criterion described in the previous section. Activation was seen in 31 of the infants; this represents $57 \%$ of all infants enrolled in the study and $69 \%$ of those from whom analyzable channels were obtained. We estimate our statistical error rate to be nine false-positive channels out of 374 analyzed, resulting in no more than six infants falsely flagged as activators (see Appendix for details).

We performed similar computations on the 10-mm channels. About $68 \%$ of the 823 channels were deemed analyzable, and $64 \%$ of the infants with analyzable channels had at least one channel pass the $t$-test for activation.

\section{Discussion}

\subsection{Infant Data Rejection Rate}

As noted in Sec. 1, most fNIRS studies of alert 6- to 9-monthold infants reject all data from about $50 \%$ of the subjects due to various acquisition flaws. While this rejection rate can be tolerable for research studies, it all but precludes clinical applications. The first goal of this study, therefore, was to examine the infant rejection rate under acquisition conditions designed specifically to reduce that rate.

Obtaining useful data involves a combination of situating fibers over the correct cortical region, achieving sufficient optical contact to interrogate that region and designing a stimulation protocol that keeps the infant calm, still, and attentive for as long as possible. There are many strategies for meeting all these needs. To achieve a low infant rejection rate, this study prioritized the infant's compliance, which led us to reduce the size of the headpiece and to increase the allure of the stimulation protocol. By reducing the number of fibers to 14 , the headpiece became quicker to fasten to the head (with just two elasticized headbands), better able to follow sudden head motion without slippage, and less distracting to the wearer, all relative to the original 34-fiber probe supplied by the vendor. As for the protocol, the addition of looming faces, more engaging rest periods, and constant music dramatically increased infant compliance compared to checkerboards and fixation crosses alone. This study narrowly defined useful (analyzable) fNIRS data as oxyhemoglobin time series with detectable heartbeats and no motion artifacts during at least two trials. These criteria rejected instances of poor fiber-tissue contact, whether due to infant motion, poor securing of the headpiece, or individual fiber contact issues due to hair or other obstructions. In essence, the selected channels were simply those where some tissue region's hemodynamics were recorded without interference from optical artifacts, permitting meaningful interpretation.

The results of this study show that it is possible to have an infant rejection rate far lower than $50 \%$ if the conditions are tailored towards that end; the rate here was $\sim 17 \%$ (9 out of 54 infants). Heartbeat was detected for every infant, meaning that the probe was always secured with sufficient optical contact for at least one channel. On average, 12 of the 20 possible channels per infant showed sufficient heartbeat. The nine rejected infants (out of 54) were all due to motion artifacts in too many trials. Given our choice of a lightweight probe able to be rapidly fastened with two single headbands, it was impossible to guard against the most noncompliant infants producing major artifacts, for example, by reaching up and touching the headpiece. More secure fastening could reduce this failure rate but might come at the expense of longer setup time, thus wasting the infant's limited attention span.

Marking a channel as analyzable did not guarantee that the tissue region it optically probed included the brain region of interest. Indeed, the large drawback of this probe design is its reduced coverage area. This created the need to place the probe precisely. In earlier data runs, the probe was centered over the inion (adapting an adult protocol); with this placement, responses to the visual stimulus were not detected. Moving the probe $2 \mathrm{~cm}$ higher ${ }^{24}$ made a crucial difference. The smaller area also prevented us from monitoring a remote region as a simultaneous control. In the future, it would be desirable to attach multiple small-area probes or a single larger-area probe without compromising infant compliance or motion artifacts.

Although the 36-mm channels were excluded from this analysis due to insufficient signal to noise for heartbeat detection, we note that some 36-mm channels exhibited clear activation-like trends. As mentioned earlier, visual activation responses are typically stronger than heartbeat, so this finding is unsurprising. There is no reason to discard such channels in general for fNIRS research. In this report, we use a conservative criterion on purpose to set a lower bound on the number of analyzable channels.

\subsection{Visual Activation Response Detection}

To our knowledge, all published fNIRS studies of alert 6- to 9-month-old infants have reported analysis on grand averages. The ability to detect hemodynamic activations in single infants would have obvious relevance to clinical applications, but to our knowledge, this aim has not been directly addressed in the literature for this age group. A secondary goal of this work, therefore, was to measure stimulus-related activations 
in individual alert 6- to 9-month-olds and report the percentage of "responders." Of the 45 infants from whom analyzable channels were obtained, approximately two-thirds showed significant stimulus-correlated oxyhemoglobin surges in response to visual stimulation. This is likely a conservative estimate; even though the placement technique relative to the inion was refined for all the infants in our study, the probe due to its size may not always have overlapped with the infant's visual cortex. ${ }^{31,32}$

Larger-area probes or better guidance from anatomical landmarks (or prior structural MRI) would presumably reduce such placement errors. In addition, the analysis made no distinction regarding whether the infant was paying attention to the screen displaying the stimulus. Although video recordings were obtained from the side of the infant, the angle and dimness made it difficult to determine the location of the infant's gaze. While our rejection of motion artifacts probably also rejected many trials in which the infant was inattentive to the stimulus, a robust exclusion of nonattentive trials, either by eye-tracking or other video recording, would be desirable. If placement errors were eliminated and trials of infant nonattentiveness were discarded, we predict that the percentage of infants displaying activation responses would rise noticeably.

An interesting finding was that activation signals were detected over the occipital cortex by some of the $10-\mathrm{mm}$ source-detector separation channels. Typically, 10-mm channels are considered short enough to treat as "scalp-only" channels for subtraction of superficial hemodynamic interference. Here, however, we observed stimulus-correlated increases in oxyhemoglobin concentration directly in the $10-\mathrm{mm}$ data from $64 \%$ of the infants, applying the same criteria as for the longer channels (22 and $30 \mathrm{~mm}$ ).

This implies that the 10-mm channel may have been sensing cortical hemodynamics. Taga et al. ${ }^{20}$ reported a similar conclusion when using a 10-mm source-detector separation distance, and Brigadoi and Cooper $^{33}$ have recently drawn similar conclusions about optimal short distances for correction from Monte Carlo simulations. However, an alternative explanation is that a stimulus-correlated hemodynamic response was present in extracerebral tissue (i.e., scalp). Further experiments are intended to explore the nature of short-separation channels with control measurements over other brain regions, especially pertaining to the potential for removal of superficial hemodynamics.

\section{Conclusion}

$100 \%$ of the infants in this study yielded channels that exhibited heartbeat. About $83 \%$ of these infants produced analyzable channels, with the data dropout due to motion artifact rejection. Finally, $69 \%$ of the infants were flagged as showing significant hemodynamic activations. Beyond reporting activation results for infants in the 6- to 9-month age range, this study outlined several methodological adjustments that were important for obtaining such data. That is, a lightweight comfortable probe, stimulus and rest protocol that maximizes attention, and adequate optical contact are essential for acquiring individual infant time courses. Consequently, these individual time courses, whether they are further averaged in a group dataset or analyzed singularly, can be applicable in either a research or a clinical setting, with increased confidence in the quality of the data.

\section{Appendix: Estimation of the False Positive Rate}

Infants were flagged as activators if at least one fNIRS channel passed the $t$-test described in Sec. 3.3. For some infants, only one channel passed; for others, many channels passed. Analysis was performed to estimate how many infants would have been flagged in this fashion due to noise alone. In other words, the false-positive rate due to multiple comparisons was estimated.

To start with, 11 infants had exactly one channel $(n=1)$ flagged. These 11 infants had a total of 97 analyzable channels (those that have passed both the heartbeat and motion artifact criteria) and as such were considered for the $t$-test. Under the approximation that all channels had independent noise, the expected number of false positives (using $p=0.05$ ) is 5 . In the most pessimistic scenario in which all five of these channels are from different infants, then five infants would be falsely flagged.

We repeated this analysis for the group of infants that had $n=2$ channels flagged, then $n=3$, and so on. For the $n=3$ cohort, four false positives were predicted; if those happened to cluster, they could account for one infant being falsely flagged. No other cohort with $n>1$ showed a statistical likelihood of having an infant with that many falsely flagged channels. Hence, the total number of false-positive babies from this analysis is six: five from $n=1$ and one from $n=3$.

\section{Acknowledgments}

Funding from the National Science Foundation (Grant No. CBET-0931687) and the National Institutes of Health (Grant No. HD-037082) is gratefully acknowledged. In addition, funding from the Australian Commonwealth Government (Australian Postgraduate Award) and Queensland University of Technology is gratefully appreciated. Finally, we thank Johnny Wen for help with the code for the stimulus presentation.

\section{References}

1. J. H. Meek et al., "Regional hemodynamic responses to visual stimulation in awake infants," Pediatr. Res. 43(6), 840-843 (1998).

2. S. Lloyd-Fox, A. Blasi, and C. Elwell, "Illuminating the developing brain: the past, present and future of functional near infrared spectroscopy," Neurosci. Biobehav. Rev. 34(3), 269-284 (2010).

3. A. Cristia et al., "An online database of infant functional near infrared spectroscopy studies: a community-augmented systematic review," PLoS One 8, e58906 (2013).

4. D. C. Hyde et al., "Near-infrared spectroscopy shows right parietal specialization for number in pre-verbal infants," Neuroimage 53(2), 647-652 (2010)

5. Y. Otsuka et al., "Neural activation to upright and inverted faces in infants measured by near infrared spectroscopy," Neuroimage 34(1), 399-406 (2007).

6. H. Watanabe et al., "Functional activation in diverse regions of the developing brain of human infants," Neuroimage 43(2), 346-357 (2008).

7. T. Wilcox et al., "Hemodynamic changes in the infant cortex during the processing of featural and spatiotemporal information," Neuropsychologia 47(3), 657-662 (2009).

8. T. Wilcox, J. A. Haslup, and D. A. Boas, "Dissociation of processing of featural and spatiotemporal information in the infant cortex," Neuroimage 53(4), 1256-1263 (2010).

9. R. N. Aslin, "Questioning the questions that have been asked about the infant brain using near-infrared spectroscopy," Cognit. Neuropsychol. 29(1-2), 7-33 (2012). 
10. R. N. Aslin, M. Shukla, and L. L. Emberson, "Hemodynamic correlates of cognition in human infants," Ann. Rev. Psychol. 66(1), 349-379 (2015).

11. R. E. Vanderwert and C. A. Nelson, "The use of near-infrared spectroscopy in the study of typical and atypical development," Neuroimage 85(Pt 1), 264-271 (2014).

12. T. Wilcox et al., "Infants' scanning of dynamic faces during the first year," Infant Behav. Dev. 36(4), 513-516 (2013).

13. H. Ichikawa et al., "Contrast reversal of the eyes impairs infants face processing: a near-infrared spectroscopic study," Neuropsychologia 51, 2556-2561 (2013).

14. Y. Otsuka et al., "Eye contrast polarity is critical for face recognition by infants," J. Exp. Child Psychol. 115(3), 598-606 (2013).

15. A. Hirshkowitz and T. Wilcox, "Infants ability to extract three-dimensional shape from coherent motion," Infant Behav. Dev. 36(4), 863-872 (2013).

16. T. Wilcox et al., "Cortical activation to object shape and speed of motion during the first year," Neuroimage 99, 129-141 (2014).

17. T. Wilcox et al., "The effect of color priming on infant brain and behavior," Neuroimage 85(Pt 1), 302-313 (2014).

18. D. A. Boas et al., "Twenty years of functional near-infrared spectroscopy: introduction for the special issue," Neuroimage 85( $\mathrm{Pt} 1), 1-5$ (2014).

19. B. W. Zeff et al., "Retinotopic mapping of adult human visual cortex with high-density diffuse optical tomography," Proc. Natl. Acad. Sci. 104(29), 12169-12174 (2007).

20. G. Taga, F. Homae, and H. Watanabe, "Effects of source-detector distance of near infrared spectroscopy on the measurement of the cortical hemodynamic response in infants," Neuroimage 38(3), 452-460 (2007).

21. J. Gervain et al., "Near-infrared spectroscopy: a report from the medonnell infant methodology consortium," Dev. Cognit. Neurosci. 1(1), 22-46 (2011).

22. N. Tottenham et al., "The nimstim set of facial expressions: judgments from untrained research participants," Psychiatry Resident 168(3), 242-249 (2009).
23. N. Z. Kirkham, J. A. Slemmer, and S. P. Johnson, "Visual statistical learning in infancy: evidence for a domain general learning mechanism," Cognition 83(2), B35-B42 (2002).

24. C. Kabdebon et al., "Anatomical correlations of the international 1020 sensor placement system in infants," Neuroimage 99, 342-356 (2014).

25. J. R. Goodwin, C. R. Gaudet, and A. J. Berger, "Short-channel functional near-infrared spectroscopy regressions improve when sourcedetector separation is reduced," Neurophotonics 1(1), 015002 (2014).

26. F. F. Jobsis, "Noninvasive, infrared monitoring of cerebral and myocardial oxygen sufficiency and circulatory parameters," Science 198(4323), 1264-1267 (1977).

27. S. Brigadoi et al., "Motion artifacts in functional near-infrared spectroscopy: a comparison of motion correction techniques applied to real cognitive data," Neuroimage 85(Pt 1), 181-191 (2014).

28. T. Huppert et al., "Homer: a review of time-series analysis methods for near-infrared spectroscopy of the brain," Appl. Opt. 48(10), D280-D298 (2009).

29. R. B. Saager, N. L. Telleri, and A. J. Berger, "Two-detector corrected near infrared spectroscopy (C-NIRS) detects hemodynamic activation responses more robustly than single-detector NIRS," Neuroimage 55(4), 1679-1685 (2011).

30. Student, "The probable error of a mean," Biometrika 6(1), 1-25 (1908).

31. L. L. Emberson, J. E. Richards, and R. N. Aslin, "Top-down modulation in the infant brain: learning-induced expectations rapidly affect the sensory cortex at 6 months," Proc. Natl. Acad. Sci. 112(31), 9585-9590 (2015).

32. S. Lloyd-Fox et al., "Coregistering functional near-infrared spectroscopy with underlying cortical areas in infants," Neurophotonics 1(2), 025006 (2014).

33. S. Brigadoi and R. J. Cooper, "How short is short? Optimum sourcedetector distance for short-separation channels in functional near-infrared spectroscopy," Neurophotonics 2(2), 025005 (2015).

Biographies for the authors are not available. 